\title{
Using Software Agents to improve functionality of the Power Quality Management Software System
}

\author{
Dan Apetrei ${ }^{1}$, Ralf Neurohr ${ }^{2}$, Mihaela $\mathrm{Albu}^{2}$, Valentin Rascanu ${ }^{2}$, Petru Postolache ${ }^{2}$, Nicolae Golovanov ${ }^{2}$, and \\ Ioan Silvas ${ }^{1}$ \\ ${ }^{1}$ SC Electrica SA, str. Grigore Alexandrescu nr. 9, Bucharest, 011621 Romania; e-mail: \\ dan.apetrei@electrica.ro;ioan.silvas@electrica.ro \\ 2 "Politehnica" University of Bucharest, Splaiul Independenţei nr.313, Bucharest 060042 Romania e-mail: \\ ralf.neurohr@gmail.com, albu@ieee.org, vrascanu@yahoo.com, postolachepetru@yahoo.com, \\ nicolae_golovanov@yahoo.com,
}

\begin{abstract}
Power quality management is a complex problem that involves rapid changes in knowledge management. That's why hardware and software systems have to continuously adapt the dynamic requirements of the customer and to process increasing quantities of data. Software Agents could be of help in developing a self adapting dynamic system.
\end{abstract}

\section{Key words}

Power quality management, Software Agent,

\section{Introduction}

Software agents are developed under the concept of multi-agent systems (MAS). A simple search on Google [1] for the number of references on this subject and their evolution in time, gives the results presented in the chart in fig. 1-1.

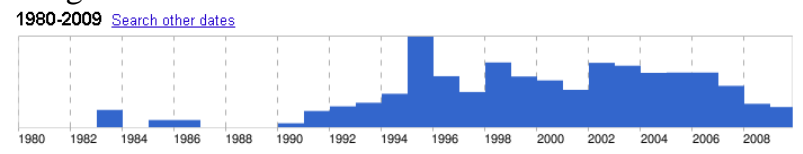

Fig. 1-1 evolution of MAS web pages

Last decade power engineering domain was rich in communication regarding MAS application [2]. IEEE transactions and Conference papers reported a broad range of applications like: diagnostics [3]; monitoring [4]; power system restoration [5]; market simulation [6,7]; network control and automation [7,8,9]. The technology is getting mature enough to go from the laboratory to the utility, allowing industry to evaluate MAS effectiveness [3].

Our paper evaluates the impact of MAS technology on PQMSS (power quality management software system) by describing main areas of PQMSS and showing immediate application areas. First part of the paper is a short presentation of the important PQMSS components. Second part gives a short description of software agents and their uses in industry. In the end ways to improve PQMSS using software agents are identified and presented. The use of software agents is evaluated over the data, information, knowledge pyramid.

\section{PQMSS aspects}

Following paragraphs shortly describe important aspects of PQMSS. First we give a short note on network model from database point of view, then we discuss hardware and software levels.

\section{$2.1 \quad$ Power quality Network level model}

Most of the cases a distribution network, could be abstracted from power quality recorded events point of view as a tree presented in fig. 1-2.

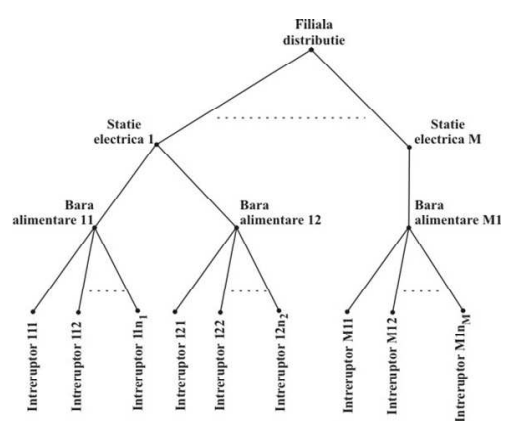

Fig. 2-1 tipical distribution network topology

This simple way to describe network is good enough to provide the support for most of the network aggregated quality parameters calculation. That's why the database structure should be mapped on a structure based on node and node responsible.

\subsection{Hardware system}


First stage of PQMSS is hardware. Nowadays, all data sources are more or less integrated in a common data repository in order to support decision. Primary data collecting equipment usually has a configuration similar to the one presented in fig. 1-3, or at least includes modules similar to the ones presented in the chart.

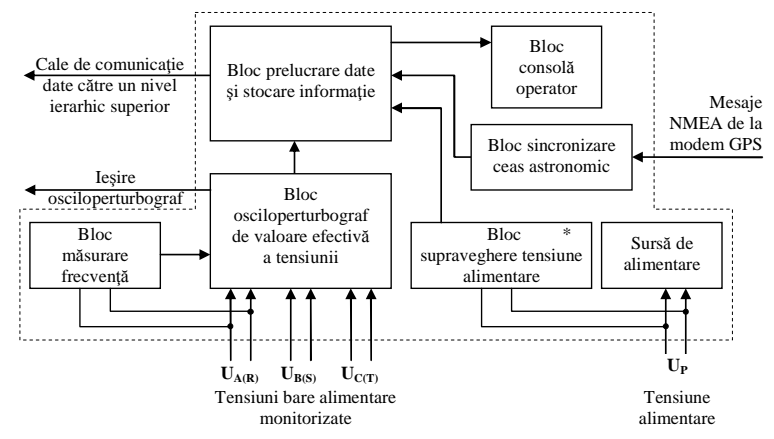

Fig. 2-2 - typical hardware for local data acquisition

The architecture presented in fig. 1-3 is typical for portable equipments. If we intend to get a stationary hardware for network nodes like the ones presented in fig. 1-2 it is rational to extend the architecture in fig. 1-3 by elements that could increase module versatility as could be seen in fig. 1-4.

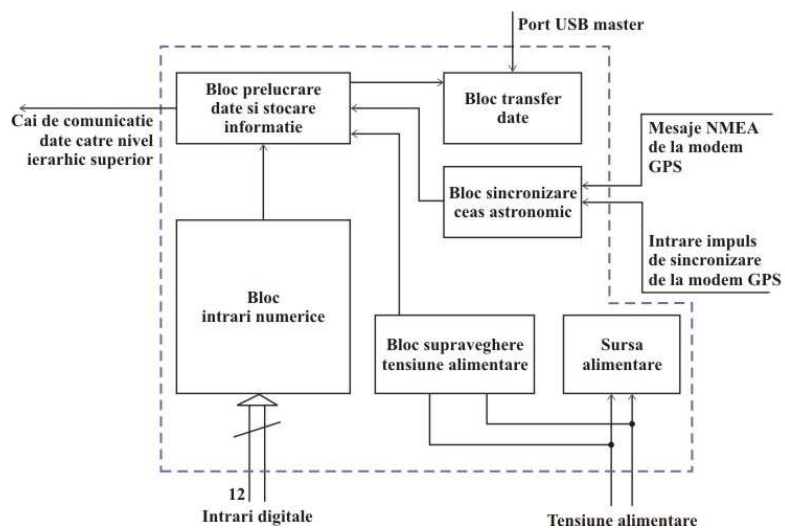

Fig. 2-3 extension solution for local hardware system

The extension of local systems is based on the fact that the voltage would be the same for all the branches getting from a common node. Next level of hardware integration would be related to the first survey/decision hop. Fig. 1-5 presents two common architectures for most of the systems.

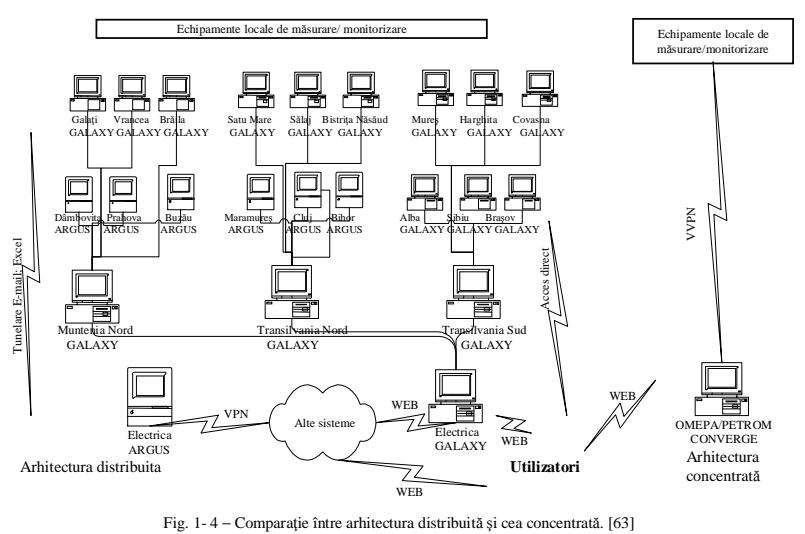

Fig. 2-4 survey/decision level hardware integration [10]
The left part of the drawing shows a distributed system, while the right part shows a concentrated one. Criteria for choosing one architecture or another are:

- The way data base management system is accessed;

- Administration and configuration requirements;

- Speed of the system correlated to decision agility;

Of course among decision criteria there are also non-technical factors like: cost of promoting the investment; human resources competencies availability.

\subsection{Software level}

Layered model of software development is presented in fig. 1-6. The drawing shows the layers included in local machines like: firmware, middleware or operating system and the way it collaborates with external layers in order to integrate each other.

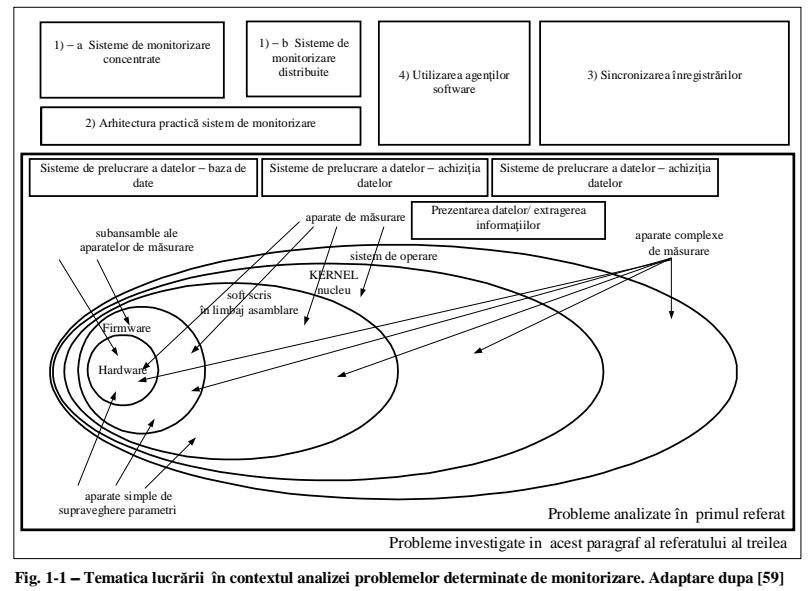

Fig. 2-5 software layers of PQMSS [11]

Fig. 1-7 shows a practical approach of software layers implementation and data integration with relevant information sources.

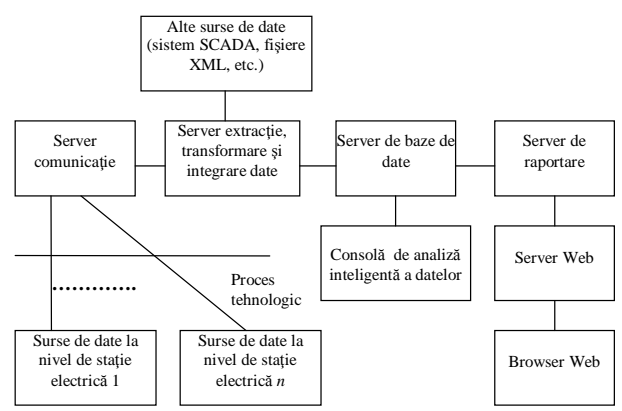

Fig. 2-6 Overall software architecture

As could be seen in fig. 1-7 there are multiple servers that have to interact in order to fulfil PQMSS functions. The servers presented in the figure are: communication server; data extraction server; database server; reporting server; web server. The system presented in fig 1-6 could be one of the data sources in fig. 1-7.

\section{Data - information - knowledge pyramid}


Most of the software systems aims to support decision. PQMSS is no exception from this empirical rule. Without informed decisions there is scarce improvement in Power quality. Fig. 2-1 shows the data, information, knowledge concept like a pyramid.

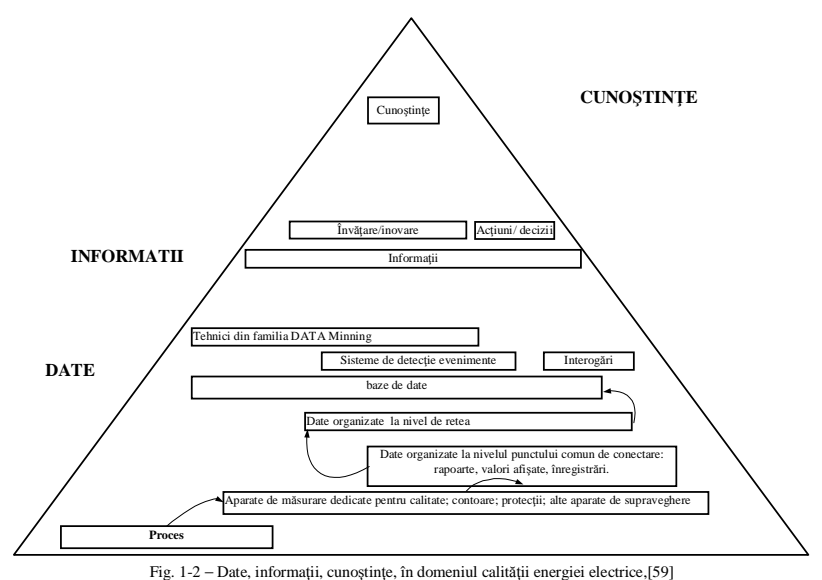

Fig. 3-1 data information knowledge [11]

Data is the result of most of the measurement processes. Using equipments like the ones described in paragraph 1.2 we get data from the process and organize it in a database. Next level would be information that comes from an interrogation launched on the database. Usually the information that we got is meant to support some decision. The more information we get, the more learning process could start. The result of the learning process could be sometimes knowledge.

\section{Software Agents}

Software agent is a computer program that interacts with the user or other program similar to an agency. This implies at agent level the authority to decide the appropriate action and the moment when it should be launched. On short the agent is not called like a normal task, but is automated activated.

The concept is related to object oriented programming as long as an agent has: methods, functions and objects. This way is handy to describe a software entity that is capable of acting with a certain degree of freedom in order to conclude some task as the user would do. Classical definition of the agent [13] states that the agent is hardware or software entity placed in an environment to witch is capable to interact independent/autonomous in order to change it. Everything that is external to the agent is considered environment. In order to be able to interact with the environment, the agent has to be capable to observe or change the environment. For a physical environment as in the power system, the observability could be fulfilled through sensors. If the environment is a virtual one, based on mathematical models, as in computing systems, the observability is determined by calls to system routines, dedicated programs or messaging systems. The way an agent changes the environment could be reflected in the hardware. For instance within distribution network a breaker is opened. One example of reflecting in software the change determined by an agent is for instance the storage of diagnoses data in a database.

Since the agent could be separated from the environment it could be distributed also. Copies of the same agent placed in different environment conditions would not react the same. The capability of an agent to process date and produce decision should be unaffected. Most probable, depending on context, the action take by an agent would differ from case to case. This means that an agent could be useful in any environment/context that accepts actions witch agent was meant to fulfil.

Agent definition [13] also asks for autonomy. The autonomy concept is vague and difficult to define. One of the less restrictive definitions of the autonomy of an agent is the one that states that it is permitted to an agent to have control of its own actions [14]. That means the agent could initiate or program for execution some particular action. One step ahead in defining autonomy is that the agent should launch an action based on environment change not based on knowledge gathered.

There are cases when these definitions are useless in discriminating actual systems from agent based ones. For instance, relay protection system could be considered as agent based system since:

- It works in an environment - the power system;

- Reacts to the changes of the environment as in changes of the current or voltage parameters;

- It has a certain degree of freedom.

Similar analysis could be done for unix daemons or virus protection systems.

Anyway just renaming existing systems does not lead to any benefit. That's why it is necessary to preciselly identifying advantages and disadvantages of the agent based systems. As a matter of fact, these differences are the reason why agents are promoted in solving problems within power network. Main reason to use an agent is its intelligence that it gets by extending the autonomy concept to flexible autonomy $[13,14]$. Coming from this there are three characteristics of an agent: reactivity; getting pro-active; developing social abilities. Since an agent is reacting to environment changes this is a proof of reactivity. Pro-active behaviour is determined by the way a decision is adopted based on objective. This implies behaviour changes to reach the goal. For instance if an agent looses the link with the agents that had to provide some services to fulfil its task, then the agent looks for another supplier of the service required.

Social abilities are determined by the capability of an intelligent agent to interact with another intelligent agent. Interacting is more than transfer of data between entities. The main feature of interaction is the ability to negotiate in order to cooperate. Nowadays this ability is supported by a communication language named ACL (agent communication language) [15]. Social abilities make obvious the need for a MAS environment that is composed by two or more agents. Opposite to objects that are defined by methods and attributes, the agents are defined by behaviour [16,17].

Fig. 4-1 presents a classification of agent's type based on: scope, motivation, roles, potential benefits. Based on cooperation, learning or autonomy capabilities concepts balance, the agents could be: learning collaborative, 
collaborative or interface. Core of the concept is the smart agent that has features from all the concepts discussed.

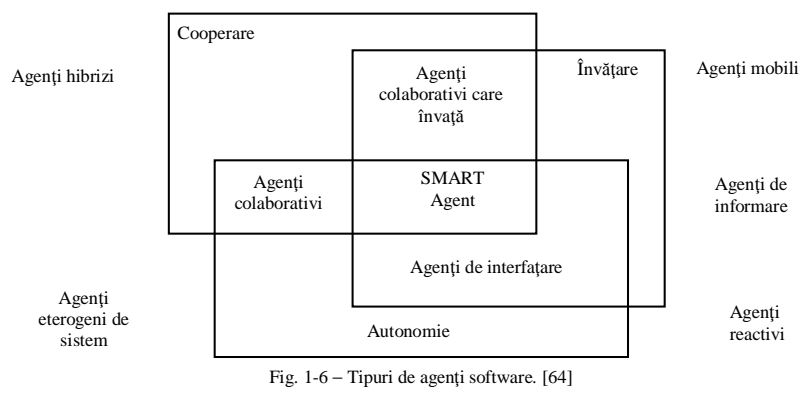

Fig. 4-1 Type of Software agents

For PQMSS software agents are detecting, anticipating faults or network monitoring [18].

Using agents is favoured by the complex landscape of power systems nowadays. We are now experiencing multiple responsible parties for distribution, transport, supply or balancing. Even if these complex relations could be modelled, multiple changes that are going to take place would trash the model.

\section{Software agents application within PQMSS}

One of the complex aspects in power quality monitoring is data processing and presentation. Besides the hurdles determined by the ambiguity of the quality problems, there is also a complex distribution routine of the quality reports.

PQMSS could benefit from agent technology by developing the automate user/primary monitoring equipment interface. Instead of user accessing PQMSS, we get an active system keeping user informed as needed. The advantages are obvious in terms of time and processing power consumption on servers and user commodity.

Agents are user configurable and could be updated by a learning process based on user reports.

The idea is to install two classes of agents (interface agents and informative agents) over the architecture presented in fig. 2-6.

The interface agents would deals with:

- Link between communication server and extraction server;

- Protocol between database server and extraction server;

- Dialogue between database server and reports server;

Once the report stimulus is activated, (could be a trigger based on time scheduler or a trigger based on thresholds), agents chain is launched in order to initiate the report sequence from communication server to database server through extraction server for the reports server.

The second class of agents (informative ones) are gathering the information from interface agent's work and opening communication channels to the user. Scenarios like content delivered to the user on the active channel could be handled by the informative agent. Besides that, the informative agent could initiate a collaboration process with similar agents in the system in order to increase information content of the report.

Depending on the report content, agent has to decide the destination user class between: transport/distribution operator, supplier or client. If the events are affecting the functionality of the system on list of beneficiaries PQMSS administrator could be included.

\subsection{Practical consideration}

Content of this paragraph is based on practical field test of hardware and software components of Electrica's PQMSS pilot. As test confirguration the system uses combined web (Apache1) and database (MySQL2) server architecture, driven a business logic written in PHP3. Both servers and the script interpreter being available for different OS platforms.

The software can be run locally on a dedicated machine, as an intranet application in a local network or remotely as an internet application because archive upload is also supported. It is even possible to run the complete system from an USB flash drive for demonstration purposes. Moreover, the system's design is also supporting the direct call of business logic programs from the OS console. This is an efficient way to process complete archives directly in an automated way. That part of the system is more or less agent based as in a MAS environment dealing with the multiple data collection nodes. This is core of the system, regarding the first step of making the data accessible for the human operator without the consumption of human resources.

For local operation all software components and servers have to be installed on a single computer which does not need any network connection. Installation and configuration will not consume significant effort and time if a pre-installed / pre-configured server system like XAMP4 is used.

The distribution of data comes with the web server and database server in a special server directory tree providing also the well known phpMyAdmin5 toolbox for the administration of the database server.

Both, intra- and internet installations are very similar and they should be planed and accomplished with the network or server administrator. Security issues will be less important for the intranet solution, but still is recommended not to allow unlimited access for all users to the application directory since the upload feature, which supports the upload of single data files and complete archives, might be abused.

A typical intranet installation would for instance provide a monitoring database, which is collecting long term power quality data from different nodes in a grid for the generation of analytical reports for the grid and studying eventual propagations of "events". Because of its high scalability, such a system would combine theoretically unlimited storage capacity with a high availability of information. The main differences in alternative implementations will be related to the mechanisms for feeding the database:

- Supposing a powerful system, running at rather low loads, archive files can be put by selected 
users directly into the servers upload directory and the feeding process can be started by calling batch processing console scripts remotely.

- If the system gets overloaded by the former method, an additional dedicated stand alone system should be considered for the processing of archives.

The processing results can be written directly into the database or stored in an intermediate database which will inject single records via SQL statements during low load periods of the server. That is already software agent job.

In the current version the database is providing only 2 tables, one of them serving as an archive which is providing the complete original data in compressed form plus a quick access to some summarising statistical information and graphical plots for voltage, frequency and their first numerical derivatives.

The second table is holding exclusively the summarising statistical information in a modified way, which is providing faster and better access for the efficient generation of reports, overviews and the detection of "events" and critical situations. Both tables are linked by an unique key, which is assuring the database integrity and avoids the insertion of duplicate or ambiguous records. Processing of data from one table to another is done through software agents for every node feeding the database.

User interface is an interactive web page, which was the starting point of the whole development. It currently supports the following functions, which were added step by step during the ongoing analysis of the boundary conditions and the requirements for a meaningful statistical analysis of and the data aggregation for the elementary 10 minutes recordings:

- Opening and processing of a single 10 minutes CSV repository.

- Opening and presenting of a complete archive containing a variable number of compressed CSV repositories, including the sorting of the repositories by their time stamps.

- Selection and processing of individual CSV repositories from an archive.

- Estimation of some basic statistical measures of location and dispersion for voltages, half cycle durations and the derived frequencies, including averages, minima, maxima and ranges 1.

- Estimation of some basic statistical measures of location and dispersion for the first numerical derivatives of voltages, half cycle durations and frequencies, including averages, minima, maxima and ranges.

- Generation of line plots for voltages and frequencies and their first numerical derivatives.

- Optional storage of the time stamps, the estimated basic statistical measures, the compressed raw data and the generated plots in the archive database.

- Upload of data files from a remote site for processing them on the server. Currently this feature works for single CSV repositories or smaller archives. The common archives for one week, holding about $1000 \mathrm{CSV}$ repositories with an equivalent of 100 to $140 \mathrm{MB}$, will be rejected by the standard settings for upload file size restrictions on most web servers.

The best description for this web page from the users point of view would be "the elementary time interval inspector" while it can be seen with the eyes of a developer as the "cradle of the system's development and evolution". In fact, the script is nothing else than an extended, but silent version of the user interface.

Despite the fact that this part is not developed in an agency filosophy yet, it is expected to take the chance for the next revision.

\section{Conclusion}

PQMSS is a complex problem to deal with. Most of the software, hardware aspects could benefit from software agent technology. The paper present a virtual scenario for user interface improvement using software agents.

With the practical example developed we could state that:

- The numerical and graphical comparison of the results obtained from PQMSS pilot and those which are generated by StatSoft's STATISTICA are clearly demonstrating that the numerical algorithms are working in a reliable way, producing trustable output.

This conclusion is related to data import, graph generation, numerical differentiation and the estimates for the statistical parameters. The estimation of future (not yet implemented) parameters have to be validated separately, after their implementation.

Regarding the further use of the estimated statistical parameters it has to be noticed, that the estimates are stored in the archive table as serialised objects, i.e. in a binary equivalent form with full precision. The data, which are written to statistical summary table, are stored as binary coded float types, thus supporting also data integrity and full precision. More details can be found in

\section{References}

[1] http://www.google.com/search?hl=en\&tbo=1\&tbs=tl:1\&q= $\% 22$ multi+agent+systems \%22\&ei=p9FSS6ngC4uksQac0OTY $\mathrm{Cw} \& \mathrm{sa}=\mathrm{X} \&$ oi $=$ tool\&resnum $=3 \& \mathrm{ct}=$ tlink\&ved $=0 \mathrm{CCcQpwU} \quad$ checked in January 2010

[2] Stephen D. J.McArthur, Euan M. Davidson,Victoria M. Catterson, Aris L. Dimeas, Nikos D. Hatziargyriou, Ferdinanda Ponci, Toshihisa Funabashi, - "Multi-Agent Systems for Power Engineering Applications-Part I: Concepts, Approaches, and Technical Challenges" IEEE TRANSACTIONS ON POWER SYSTEMS, VOL. 22, NO. 4, NOVEMBER 2007

[3] E. M. Davidson, S. D. J. McArthur, J. R.McDonald, T. Cumming, and I. Watt, "Applying multi-agent system technology in practice: Automated management and analysis of SCADA and digital fault recorder data," IEEE Trans. Power Syst., vol. 21, no. 2, pp. 559-567, May 2006.

[4] S. D. J. McArthur, S.M. Strachan, and G. Jahn, "The design of a multiagent transformer condition monitoring system," IEEE Trans. Power Syst., vol. 19, no. 4, pp. 1845-1852, Nov. 2004.

[5] T. Nagata and H. Sasaki, "A multi-agent approach to power system restoration," IEEE Trans. Power Syst., vol. 17, no. 2, pp. 457-462, May 2002.

[6] S. E.Widergren, J. M. Roop, R. T. Guttromson, and Z. Huang, "Simulating the dynamic coupling of market and 
physical system operations," in Proc. IEEE Power Eng. Soc. General Meeting, 2004, Jun. 2004, pp. 748-753.

[7] D. Koesrindartoto, S. Junjie, and L. Tesfatsion, "An agentbased computational laboratory for testing the economic reliability of wholesale power market designs," in Proc. IEEE Power Eng. Soc. General Meeting, 2005, Jun. 2005, pp. 931936.

[8] A. L. Dimeas and N. D. Hatziargyriou, "Operation of a multi-agent system for microgrid control," IEEE Trans. Power Syst., vol. 20, no. 3, pp. 1447-1455, Aug. 2005.

[9] D. P. Buse, P. Sun, Q. H. Wu, and J. Fitch, "Agent-based substation automation,” IEEE Power Energy Mag., vol. 1, no. 2, pp. 50-55, Mar./ Apr. 2003.

[10] Ion Lungu, Dan Apetrei, - "Unbundling implies Money Chain Changes in SM\&AB1 Systems", - Utilities Smart Metering and Advanced Billing Forum London, UK, 17th-18th January 2008

[11] Dan Apetrei,, - "Evaluarea erorilor in prelucrarea semnalelor perturbate", - UPB -referat intocmit in cadrul programului de pregatire doctorala , 2008

[12] Dan Apetrei,, - "Probleme actuale de masurare in evaluarea calitatii energiei electrice", - UPB -referat intocmit in cadrul programului de pregatire doctorala , 2007

[13] M. Wooldridge,G. Weiss, - "“Intelligent Agents" in Multi-agent Systems", - Cambridge, MA: MIT Press, Apr. 1999 , 1999

[14] S. Franklin,A. Graesser, - "Is it an agent or just a program?", - 3rd Int.Workshop Agent Theories, Architectures, and Languages 1996, http://ccrg.cs.memphis.edu/assets/papers/ Is $\% 20$ it $\% 20$ an $\% 20$ Agent, $\% 20$ or $\% 20$ just $\% 20$ a $\% 20$ Program $\% 2$ 0-\%20A\%20 Taxonomy.htm , 1996

[15]SC00061 - FIPA ACL Message Structure Specification",http://www.fipa.org/repository/standardspecs.html ,

[16] Nick Jennings ,Michael Wooldridge, - "Software Agents", - IEE Review, January 1996, pp 17-20, 1996

[17] Jeffrey M. Bradshaw,, - "Software Agents", - AAAI Press; illustrated edition edition (April 18, 1997) ISBN-10: 0262522349,1997

[18] Li Liu,Logan, K.P.; Cartes, D.A.; Srivastava, S.K., "Fault Detection, Diagnostics, and Prognostics: Software Agent Solutions", - Vehicular Technology, IEEE Transactions onVolume 56, Issue 4, July 2007 Page(s):1613 - 1622 , 2007 Digital Object Identifier $\quad$ 10.1109/TVT.2007.897219 